\title{
Schwerpunkt: Entscheidung unter Unsicherheit
}

\author{
Annette Scheunpflug/Hans-Peter Blossfeld
}

\section{Editorial}

Die Begriffe der „Entscheidungs- und Handlungsunsicherheit" haben in den Nachbarwissenschaften der Pädagogik, wie etwa der Wirtschaftswissenschaft, der Soziologie und der Psychologie, in den letzten Jahren eine steile Karriere gemacht. In der Ökonomie hat man das ursprüngliche Modell des voll informierten, mit geordneten Präferenzen und unbegrenzter Rechenkapazität ausgestatteten Homo oеconomicus zunehmend aufgegeben zugunsten eines Modells des Individuums, das unsicher ist, weil es unvollständig informiert ist, Zielkonflikte hat und unter Zeitdruck „,vernünftige“ Entscheidungen fällen muss. In der Soziologie wird in den neueren Rational-Choice-Ansätzen zunehmend der Versuch unternommen, den Homo oeconomicus mit dem Homo sociologicus zu verbinden, so dass deutlich wird, dass soziale Normen und moralisches Handeln durchaus einen fundamentalen ökonomischen Sinn haben. Moralische Kategorien wie Ehrenhaftigkeit, Akzeptanz von Autorität oder Vertrauen reduzieren beispielsweise Transaktionskosten und Unsicherheiten aller Art beträchtlich, werden aber durch den raschen Strukturwandel zunehmend in Frage gestellt. Handlungs- und Koordinierungsunsicherheit sind die Folge. In der Psychologie setzen sich zunehmend Ansätze durch, die sich auf die in der Realität bei Unsicherheit häufig anzutreffenden heuristischen Entscheidungsprinzipien (z.B. das „Satisficing“ von Herbert SIMON) oder heuristische Entscheidungsmechanismen (z.B. „fast and frugal decisions“ von GIGERENZER oder „Ecological Rationality“) konzentrieren. Allen diesen Ansätzen ist gemeinsam, dass sie sich mit den Bedingungen und Konsequenzen der scheinbar oder real zunehmenden Entscheidungs- und Handlungsunsicherheit in der modernen Gesellschaft auseinandersetzen. Eine spezifische Herausforderung des Lebens in der Postmoderne sei es - so eine häufig geäußerte Position -, Entscheidungen unter den Bedingungen von zunehmender Unsicherheit fällen zu müssen. Es sei dahin gestellt, inwiefern eine solche Zeitdiagnose zutreffend oder hinreichend plausibel ist. In jedem Fall stellt sie gegenwärtig nicht nur für die erziehungswissenschaftliche Reflexion eine Herausforderung dar, berührt sie doch zentrale Themen wie das Verhältnis von Wissen zu Nicht-Wissen, Bedingungen des Aufwachsens oder Entscheidungen über Bildungskarrieren.

Im einleitenden Stichwortartikel liefert Edwin KEINER eine wissenschaftstheoretische Grundlegung des Themas. Er beschreibt die verschiedenen Zugänge, die sich aus entscheidungs- und handlungstheoretischer, aus wissenstheoretischer sowie aus sozial- und 
gesellschaftstheoretischer Perspektive für die erziehungswissenschaftliche Reflexion von „Entscheidungen unter Unsicherheit“ ergeben. Für jeden dieser Zugänge zeigt Edwin KEINER den Nutzen für die erziehungswissenschaftliche Reflexion auf. Aus entscheidungstheoretischer Sicht kommen die Eintrittswahrscheinlichkeiten von Folgen in den Blick. Der Autor wählt als Beispiel der Umstellung auf die Output-Steuerung von Bildungssystemen und zeigt, dass in Folge dessen die Bedeutung des Pädagogischen für die Effizienz und Zielangemessenheit von Bildungssystemen sichtbar gemacht werden muss und somit ein neuer Bedarf an Wissen über Eintrittswahrscheinlichkeiten von Verhalten aufgrund pädagogischer Settings entsteht. Aus wissenstheoretischer Perspektive wird auf die Bedeutung von Wissen bzw. Nicht-Wissen verwiesen und dieses am Beispiel der disziplinären Identität der Erziehungswissenschaft verdeutlicht. KEINER zeigt am Beispiel der „Pädagogisierung“ von Diskursen Formen der Absorption von Unsicherheit auf und verdeutlicht damit abschließend exemplarisch den Nutzen sozialwissenschaftlicher $\mathrm{Zu}$ gänge für das Thema.

Unter den Bedingungen von Unsicherheit werden Bildungsentscheidungen potenziell intransparent und riskant. Steffen HILLMERT gibt mit seinem Beitrag einen Überblick über rationale Bildungsentscheidungen und deren institutionellen sowie lebensgeschichtlichen Bedingungen. Er benennt Strategiemuster, nach denen Bildungsentscheidungen unter Bedingungen von Unsicherheit gefällt werden. Dabei wird deutlich, dass die Reduktion subjektiver Unsicherheit bei Bildungsentscheidungen nicht automatisch zu einer Entscheidung zu höheren Bildungsalternativen führt.

Anja DIECKMANN und Stefan KRAUSS beschreiben Mechanismen, die aus psychologischer Sicht zur Entscheidungsfindung unter Bedingungen von Unsicherheit führen. Sie stellen die Bedeutung einfacher Heuristiken heraus, die nicht auf Wissen, sondern auf Sinneseindrücken (Blickheuristik), auf Mechanismen der Wiedererkennung (Rekognitionsheuristik) und eindimensionalen Merkmalen (One-reason decision making: Take The Best) basieren. Sie machen damit deutlich, dass Entscheidungen unter Unsicherheit nicht zwingend wissensbasiert sind und dennoch zu guten Entscheidungen führen können.

Didaktische Probleme im Umgang mit probabilistischen Entscheidungen stehen im Zentrum des Beitrags von Laura MARTIGNON und Christoph WASSNER. Ihre Untersuchungen zu einem Spiralcurriculum der Stochastik zeigen, dass Kinder bereits über Fähigkeiten im Umgang und der Nutzung von Zufällen verfügen und diese Fähigkeiten über konkrete didaktische Arrangements im Lebenslauf systematisch weiterentwickelt werden können. Damit werden Möglichkeiten für intentionale Lehrarrangements zum Lernen von Entscheidungen unter Unsicherheit angedeutet, die über die Mathematikdidaktik hinausweisen könnten.

Barbara ASBRAND untersucht mit ihrer rekonstruktiv-qualitativen empirischen Arbeit, wie Jugendliche mit Unsicherheit im Kontext der Globalisierung bzw. der Weltgesellschaft umgehen. Die Ergebnisse legen die Hypothesen nahe, dass die freiwillige Einbindung in eine Organisation Orientierungsmuster ermöglicht, die Jugendliche im Kontext der Weltgesellschaft handlungsfähiger macht. Schulische reflexiv angelegte Bildungsprozesse würden zwar zu abstrakteren Vorstellungen über die Weltgesellschaft führen, hingegen aber für den Aufbau von Handlungssicherheit weniger leitend sein.

Eine Sammelrezension zum Thema „Unsicherheit“ von Steffen HILLMERT stellt Literatur zum Thema des Heftes vor. 